\title{
EVALUACIÓN DE MATERIALES ENCALANTES Y ORGÁNICOS SOBRE LAS BASES INTERCAMBIABLES DE UN SUELO SULFATADO ÁCIDO EN INVERNADERO
}

\author{
EVALUATION OF ORGANIC MATERIALS AND LIMING ON \\ EXCHANGEABLE BASES OF AN ACID SULPHATE SOIL AT \\ GREENHOUSE CONDITIONS
}

\author{
Andrea A. Bernal ${ }^{*}$, Jenny C. Montaño², Rosaura Sánchez³ ,Yuri L. Albarrán² y Fabio E. \\ Forero $^{4}$
}

Recibido para publicación: Enero 24 de 2014 - Aceptado para publicación: Abril 15 de 2014

\begin{abstract}
RESUMEN
Los suelos sulfatados ácidos (SSA) se caracterizan por ser sistemas dinámicos con degradación química continua a causa de procesos de acidificación actual severa. Una de sus limitaciones está relacionada con la inhibición en la absorción de bases intercambiables, generada principalmente por excesos de aluminio de cambio, que afectan su productividad y limitan la disponibilidad de nutrientes para las plantas. El presente estudio evaluó en invernadero el efecto de algunas técnicas empleadas en recuperación de suelos, sobre las bases intercambiables de un SSA, con el fin de identificar metodologías para el manejo de estos suelos en Paipa (Boyacá). Se implementó un diseño experimental con 6 tratamientos y 5 repeticiones, donde se evaluó la incorporación de microorganismos eficientes, bagacillo de caña, abono verde y cal dolomita, así como la siembra de Brassica rapa L. como forraje, en los cuales se realizó la medición de bases antes y después de la siembra de Brassica oleracea var. Botrytis como cultivo indicador. Para analizar la información, se realizó una ANOVA y se aplicó prueba de comparación de promedios Tukey con confiabilidad del $95 \%$. Los resultados indicaron que la siembra de $B$. rapa L. como forraje y la incorporación de la misma como abono verde, junto con el uso complementario de cal dolomita, generaron un mayor incremento en $\mathrm{Ca}\left(18,21 \mathrm{cmol}^{\bullet} \mathrm{kg}^{-1}\right.$ y 16,61 $\left.\mathrm{cmol}_{c} \bullet \mathrm{kg}^{-1}\right)$ y $\mathrm{Mg}\left(6 \mathrm{cmol}_{c} \bullet \mathrm{kg}^{-1}\right.$ y $\left.5,15 \mathrm{cmol}_{\mathrm{c}} \bullet \mathrm{kg}^{-1}\right)$. El K presentó el valor más alto con la incorporación de abono verde $\left(1,31 \mathrm{cmol}_{\mathrm{c}} \bullet \mathrm{kg}^{-1}\right)$, respecto a las metodologías evaluadas.
\end{abstract}

Palabras clave: Brassica rapa, Brassica oleracea, cal dolomita, fuentes orgánicas, recuperación.

\begin{abstract}
Acid sulphate soils (ASS) are characterized to be dynamic systems with a continuous chemical degradation processes due to current severe acidification. One of their limitations is related to the inhibition of the absorption of exchangeable bases, generated mainly by excess aluminum rates, which affect their productivity

\footnotetext{
1*Bióloga. M.Sc. (c) Ingeniería Ambiental. Grupo de Investigación en Desarrollo y Producción Agraria Sostenible (GIPSO), Universidad Pedagógica y Tecnológica de Colombia. Tunja, Colombia. Calle 42A No. 1C-12 Tunja (Boyacá) 3144864851. anbernal@gmail.com.

${ }^{2}$ Bióloga. M.Sc. Ingeniería Ambiental. Grupo de Investigación en Desarrollo y Producción Agraria Sostenible (GIPSO), Universidad Pedagógica y Tecnológica de Colombia. Tunja, Colombia.

${ }^{3}$ Ingeniera Agrónoma. M.Sc. (c) Ingeniería Ambiental. Grupo de Investigación en Desarrollo y Producción Agraria Sostenible (GIPSO), Universidad Pedagógica y Tecnológica de Colombia. Tunja, Colombia.

${ }^{4}$ Ingeniero Agrónomo. M.Sc. Ciencias Agrarias, Docente FACIAT, Grupo de Investigación en Desarrollo y Producción Agraria Sostenible (GIPSO), Universidad Pedagógica y Tecnológica de Colombia. Tunja, Colombia.
} 
and limit the availability of nutrients to plants. This study evaluated the effect of some techniques used in soil remediation on exchangeable bases of ASS, at greenhouse conditions, in order to identify methodologies for the management of these soils in Paipa (Boyacá). An experimental design with 6 treatments and 5 replications was performed, where the incorporation of efficient microorganisms, sugarcane bagasse, green manure and dolomite lime was evaluated as well as the seeding of forage Brassica rapa L.; in which the exchangeable bases were measured before and after planting Brassica oleracea var. Botrytis as indicator crop. For data analysis, was performed ANOVA and was applied Tukey comparison of means with a reliability of $95 \%$. The results indicated that the seeding of $B$. rapa as forage and incorporating of the same as green manure, with the complementary use of dolomite lime, generated a greater increase in Ca $\left(18.21 \mathrm{cmolc} \bullet \mathrm{kg}^{-1} \& 16.61\right.$ cmolc $\left.\bullet \mathrm{kg}^{-1}\right)$ and $\mathrm{Mg}\left(6 \mathrm{cmolc} \bullet \mathrm{kg}^{-1} \& 5.15 \mathrm{cmolc}^{-} \mathrm{kg}^{-1}\right)$. In terms of $\mathrm{K}$, the highest value was obtained with the addition of green manure $\left(1.31 \mathrm{cmolc}^{\bullet} \mathrm{kg}^{-1}\right)$ respect to the methodologies evaluated.

Key words: Brassica rapa, Brassica oleracea, dolomite lime, organic sources, recovery.

\section{INTRODUCCIÓN}

Los suelos se degradan normalmente por un uso no acorde con sus aptitudes y limitaciones, por prácticas de manejo agronómicas o forestales inadecuadas, contaminación industrial urbana o minera, o por fenómenos naturales como incendios y deslizamientos. Existen, además, suelos que por su origen poseen características similares a las derivadas de un proceso de degradación (Honorato y Bonomelli 2002; López 2002); dentro de las principales problemáticas que presentan se encuentra la acidificación (Urquiza 2002). Las causas de esta acidez son múltiples y entre otras se señala su origen por materiales parentales ácidos, alta percolación por excesiva pluviosidad, uso prolongado e histórico de fertilizantes de residuos ácidos (Nitrogenados, ureicos y amoniacales) y la composición de la materia orgánica, procesos que junto a otros factores contribuyen a reducir su rendimiento y producción (Ocampo et al. 2007).

La acidez en los suelos afecta de forma muy particular y determinante algunas de sus características químicas y biológicas, reduce el crecimiento de las plantas, ocasiona la disminución en la disponibilidad de algunos nutrientes como $\mathrm{Ca}^{2+}, \mathrm{Mg}^{2+}, \mathrm{K}^{+}, \mathrm{P}$ y favorece la proliferación de elementos tóxicos para las plantas como el aluminio y el manganeso (Molina 2008).

Los SSA son propios de las zonas bajas que se inundan con frecuencia o permanecen gran parte del tiempo inundados (Cabrales 2007); se caracterizan por ser sistemas con degradación química continua a causa de procesos de acidificación actual severa, debida a la toxicidad de las diversas formas reducidas del azufre, problemas como las altas concentraciones de $\mathrm{Al}^{3+}$ y $\mathrm{Fe}^{3+}$ solubles, producción de ácido sulfúrico, formación de sales o desbalanceamientos nutricionales, lo cual causa raquitismo en las plantas y atrofiamiento en su sistema radicular, que al no poder ramificarse reduce su capacidad de absorción de agua y disminuye o detiene su crecimiento (Combatt et al. 2004; GISSAT 2006; Cabrales 2007; Hernández y Castro 2007; Castro y Munevar 2011; Montaño y Forero 2013). Asimismo, la toxicidad generada en estos suelos altera su equilibrio iónico, inhibe la absorción de $\mathrm{Ca}^{2+}, \mathrm{Mg}^{2+}$ y $\mathrm{P}$ y afecta directamente su fertilidad (Castro y Munevar 2011). 
En los últimos años, muchas investigaciones se han encaminado a tratar de recuperar los suelos (Ercoli et al. 1999; González 2005; Viteri y Velandia 2006; Prasad et al. 2010), dentro de las cuales se encuentran las prácticas de manejo recomendadas para una agricultura sustentable, como el uso de residuos sobre la superficie, mantenimiento de un nivel adecuado de materia orgánica mediante la incorporación de abonos vegetales, o labranza mínima y cero, que tienden a disminuir los efectos negativos generados en los mismos (Honorato y Bonomelli 2002). De igual forma, muchas especies vegetales tienen mecanismos eficientes para la exudación de iones a través de ácidos orgánicos por las raíces, así como la capacidad de proliferación de determinadas rizobacterias y micorrizas que estimulan el crecimiento de las plantas bajo condiciones ambientales estresantes (Taylor et al. 2000).

En este contexto, la presente investigación se realizó con el fin de evaluar el efecto de técnicas empleadas en la recuperación de suelos, como la incorporación de microorganismos eficientes, bagacillo de caña, abono verde y cal dolomita, así como la siembra de B. rapa L. (nabo forrajero) como forraje (González 2005; Viteri y Velandia 2006; Molina 2008; Prasad et al. 2010) sobre las bases intercambiables de un SSA del municipio de Paipa (Boyacá), con el fin de contribuir en la búsqueda de estrategias de uso sostenible y mitigación del efecto ambiental negativo generado por estos suelos.

\section{MATERIALES Y MÉTODOS}

El SSA objeto de estudio se colectó en un lote del Sector Varguitas del municipio de Paipa (Boyacá, Colombia) a una altura promedio de $2.500 \mathrm{msnm}$, con coordenadas $5^{\circ} 4^{\prime}$ $51^{\prime \prime} \mathrm{N}$ y $73^{\circ} 3^{\prime \prime} 29^{\prime} \mathrm{W}$, debido a los reportes que se han encontrado de la existencia de suelos con características de acidez extrema y altas concentraciones de aluminio de cambio (GISSAT 2006; Dent y Dawson 2000). Adicionalmente, de acuerdo a una investigación previa se determinó que es un suelo orgánico, clasificado taxonómicamente como Hydrichaplofibrists.

Se tomó una muestra de $1 \mathrm{~kg}$ de suelo para realizar la caracterización inicial (Tabla 1), según la metodología planteada por el IGAC (2006). Asimismo, se recolectaron $450 \mathrm{~kg}$ de suelo a una profundidad no superior a $20 \mathrm{~cm}$, los cuales fueron recolectados y trasladados al Jardín Botánico de la Universidad Pedagógica y Tecnológica de Colombia (Tunja) para la realización del montaje. Se implementó un diseño experimental completamente al azar con 6 tratamientos (Tabla 2) y 5 repeticiones; cada unidad experimental (UE) estuvo compuesta por tres bolsas de polietileno para vivero, cada una con $5 \mathrm{~kg}$ de suelo, de manera que se implementó un total de 90 UE.

Para el montaje en invernadero, el suelo se sometió a desmenuzamiento manual, con

Tabla 1. Caracterización inicial de algunas sustancias químicas del suelo sulfatado ácido

\begin{tabular}{|c|c|c|c|c|c|c|c|}
\hline \multirow[t]{2}{*}{ pH } & \multicolumn{2}{|c|}{ Acidez $\mathrm{cmol} \cdot \mathbf{k g}^{-1}$} & \multirow{2}{*}{$S\left(\mathrm{mgKg}^{-1}\right)$} & \multicolumn{4}{|c|}{ Acidez $\mathrm{cmol} \bullet \mathrm{kg}^{-1}$} \\
\hline & $\mathrm{Al}^{3+}$ & A.I & & $\mathrm{Ca}^{2+}$ & $\mathbf{M g}^{2+}$ & $\mathbf{K}^{+}$ & $\mathrm{Na}^{+}$ \\
\hline 3,00 & 13,20 & 13,70 & 318 & 3,01 & 0,40 & 0,14 & 0,10 \\
\hline
\end{tabular}

ND: No determinado 
Tabla 2. Descripción de tratamientos utilizados en la investigación

\begin{tabular}{|c|c|}
\hline Tratamiento & Descripción \\
\hline $\mathrm{T} 1$ & Testigo absoluto: suelo sin aplicación de enmiendas \\
\hline $\mathrm{T} 2$ & Agronómico: 20 t•ha-1 de cal dolomita \\
\hline T3 & $\begin{array}{c}\text { Aplicación de microorganismos eficientes: } 20 \text { t• } \text { ha }^{-1} \text { de cal dolomita +inmersión de raíces del } \\
\text { cultivo indicador en microorganismos eficientes (EM) en dosificación de } 100 \text { cc en } 1 \mathrm{~L} \text { de } \\
\text { agua estéril durante } 30 \mathrm{~min}\end{array}$ \\
\hline $\mathrm{T} 4$ & $\begin{array}{l}\text { Siembra de } B \text {. rapa L. como forraje.: } 20 \mathrm{t} \bullet \mathrm{ha}^{-1} \text { de cal dolomita }+ \text { siembra de } 4 \text { semillas de B. } \\
\text { rapa L. (nabo forrajero) }\end{array}$ \\
\hline T5 & Adición de bagacillo de caña: $20 \mathrm{t} \bullet \mathrm{ha}^{-1}$ de cal dolomita $+7,5 \mathrm{t} \bullet \mathrm{ha}^{-1}$ de bagacillo de caña \\
\hline T6 & $\begin{array}{c}\text { Incorporación de B. rapa L. como abono verde: } 20 \text { t } \bullet \mathrm{ha}^{-1} \text { de cal dolomita }+4,641 \mathrm{~kg} \text { de plan- } \\
\text { tas de B. rapa L. (nabo forrajero) }\end{array}$ \\
\hline
\end{tabular}

el objeto de simular labores agrícolas de pre-siembra como rastrillada y arado. De acuerdo con los resultados obtenidos en el análisis inicial, se realizaron los cálculos de la cantidad necesaria de enmienda a emplear como correctivo, por lo cual se agregó 20 tha ${ }^{-1}$ de cal dolomita (composición promedio (\%): $55 \mathrm{CaCO}_{3}, 33 \mathrm{MgCO}_{3}, 30,8 \mathrm{CaO}, 15,7$ $\mathrm{MgO})$, a excepción de las UE del testigo. Adicionalmente, en el T5 se adicionó bagacillo de caña y en el T6 B. rapa L., en ambos casos en trozos pequeños en combinación con la enmienda calcárea; posteriormente, se procedió a realizar el montaje de las UE y se permitió un periodo de reacción de 60 días.

Al finalizar este periodo, se realizó el montaje de los demás tratamientos y la siembra del cultivo indicador, correspondiente a plántulas de coliflor (Brassica oleracea var. Botrytis) de 10 $\mathrm{cm}$ de longitud con cuatro hojas verdaderas. Se Ilevaron a cabo algunas actividades propias para algunos tratamientos, así: en el T3 Aplicación de microorganismos eficientes, se efectuó la inmersión de las raíces de 15 plántulas de B. oleracea en el biopreparado microbiano (EM), en una dosificación de 100 cc en 1 L de agua estéril durante 30 min y se sembraron en las UE del tratamiento, adicionalmente, se realizó la aplicación foliar de EM con una dosis de 30 - $40 \mathrm{~L} \mathrm{ha}^{-1}$ y se llevaron a cabo diluciones cada 45 días, a una relación de 1:10 - 20; en el T4 siembra de B. rapa L. Se sembraron 4 semillas de esta especie en cada UE, las cuales luego de 90 días se cosecharon y se sembraron en estas mismas UE las plántulas del cultivo indicador (B. oleraceae). El suelo se mantuvo bajo condiciones de humedad de capacidad de campo, para lo cual se realizó el riego con un intervalo de dos (2) días.

Al finalizar el periodo vegetativo del cultivo indicador (120 días después de la siembra), se llevó a cabo la medición de $\mathrm{Ca}, \mathrm{Mg}, \mathrm{K}$ y $\mathrm{Na}$ en el suelo, mediante el método de acetato de amonio $1 \mathrm{M}$ y neutro de acuerdo con el IGAC (2006), en el Laboratorio de Docencia de Suelos de la Facultad de Ciencias Agropecuarias de la UPTC. De igual forma, se determinó la respuesta agronómica de las plantas del cultivo indicador en cada tratamiento; la altura de las plantas se midió desde el cuello de la 
raíz hasta la hoja superior con flexómetro y el diámetro de la cabeza se midió con un calibrador Stainless Hardened. Para el análisis de la información se realizó un ANOVA con una confiabilidad del 95\% y se aplicó la prueba de comparación de promedios de Tukey con el programa estadístico SPSS $®$ versión 18.

\section{RESULTADOS Y DISCUSIÓN}

Se presentaron diferencias estadísticas significativas $(p>0.05)$ en las concentraciones de Ca, Mg y K. Los niveles de Ca evidenciaron diferencias significativas tanto entre tratamientos como en comparación con el testigo y el valor inicial (Figura 1A). El Mg presentó concentraciones superiores en los tratamientos de siembra de $B$. rapa L. $(6,00$ $\left.\mathrm{cmol}_{\mathrm{c}} \bullet \mathrm{kg}^{-1}\right)$ e incorporación de abono verde $\left(5,15 \mathrm{cmol}_{\mathrm{c}} \bullet \mathrm{kg}^{-1}\right)$, en los cuales se observó un aumento significativo con respecto a los demás tratamientos y al valor inicial (Figura 1B). Los niveles de $\mathrm{K}$ presentaron diferencias entre tratamientos y el más alto se obtuvo con la incorporación de abono verde $\left(1,31 \mathrm{cmol}_{\mathrm{c}} \bullet \mathrm{kg}^{-1}\right)$, mientras que los demás tratamientos generaron concentraciones similares entre 0,66 y $0,84 \mathrm{cmol}_{\mathrm{c}} \bullet \mathrm{kg}^{-1}$ (Figura 1C). En cuanto al $\mathrm{Na}$, aunque no se presentaron diferencias estadísticas significativas, se observó un aumentó en todos los tratamientos, excepto con la siembra de B. rapa L. el cual permaneció constante $\left(0,14 \quad \mathrm{cmolc} \bullet \mathrm{kg}^{-1}\right)$ (Figura 1D).

La incorporación de cal en el suelo contribuye y favorece el incremento de bases intercambiables, ya que la acción principal de esta técnica consiste en la corrección de las condiciones químicas del mismo; de igual forma, las prácticas de manejo que conllevan al mejoramiento químico de los SSA, deben
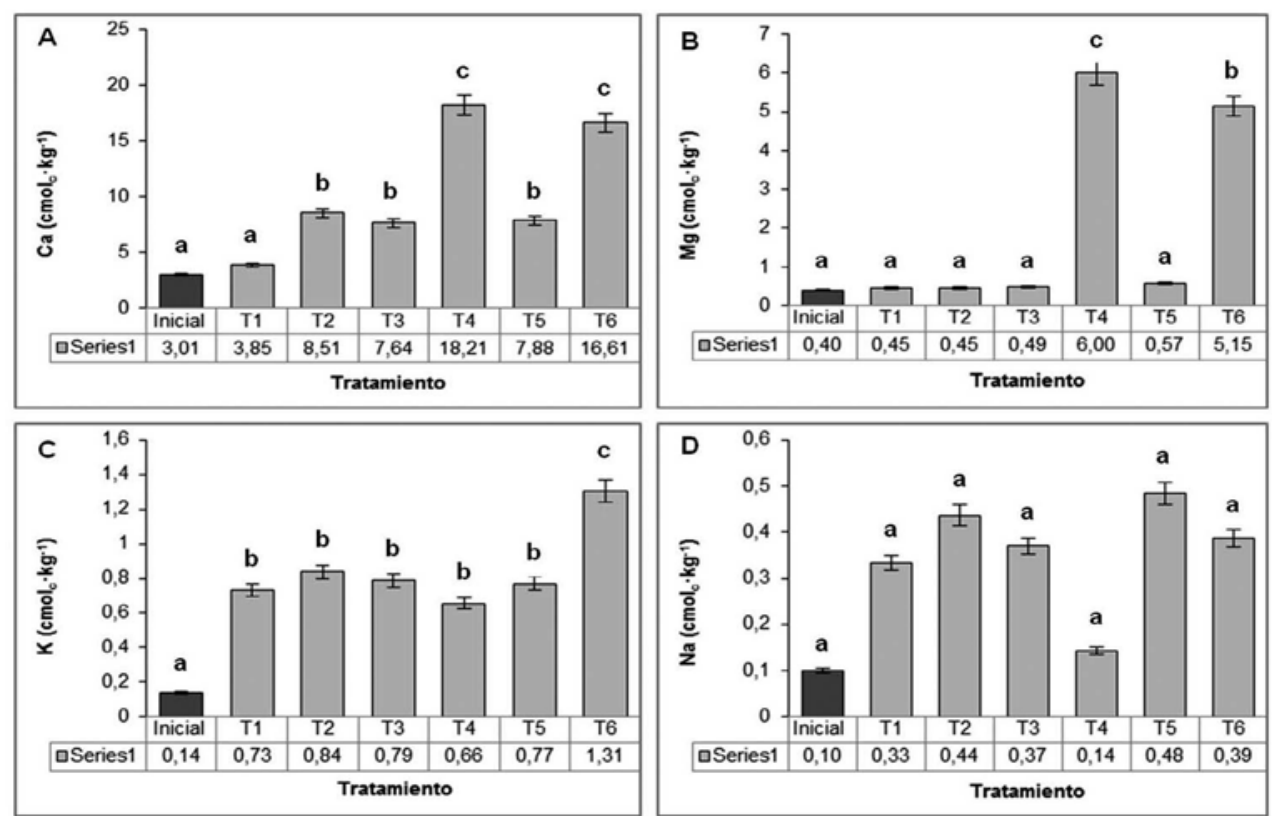

Figura 1. Evaluación de materiales encalantes y orgánicos sobre A) Calcio. B) Magnesio. C) Potasio y D) Sodio de un SSA a nivel invernadero. Inicial: valor obtenido en la caracterización inicial del suelo; T1: Testigo absoluto; T2: Tratamiento químico; T3: Aplicación de microorganismos eficientes; T4: Siembra de B. rapa L. como forraje; T5: Adición de bagacillo de caña; T6: Incorporación de B. rapa L. como abono verde. Promedios seguidos de letras distintas en la misma serie presentan diferencias significativas según la prueba de Tukey (5\%). 
contemplar el uso de materiales encalantes (Hernández y Castro 2007; Molina 2008; Castro y Munevar 2011; Osorno 2012).

El mayor incremento de Ca se generó con las técnicas de siembra de $B$. rapa L. como forraje $\left(18,21 \mathrm{cmol}_{\mathrm{c}} \bullet \mathrm{kg}^{-1}\right)$ e incorporación de abono verde $\left(16,61 \quad \mathrm{cmol}_{\mathrm{c}} \bullet \mathrm{kg}^{-1}\right)$, lo cual según Franchini et al. (1999), se debe posiblemente al efecto de sustancias exudadas por el sistema radicular del nabo forrajero, identificadas como compuestos orgánicos de bajo peso molecular, las cuales movilizan este elemento hacia la superficie. Asimismo, Plata et al. (2009) afirman que la aplicación de los mismos, en combinación con enmiendas encalantes, suministra una mayor cantidad de nutrientes debido a la naturaleza de los materiales. El tratamiento agronómico y la aplicación de microorganismos eficientes y bagacillo presentaron un aumento similar con valores entre 7,64 y $8,51 \mathrm{cmol}_{\mathrm{c}} \bullet \mathrm{kg}^{-1}$ y el testigo permaneció relativamente constante con un valor de $3,85 \mathrm{cmol}_{\mathrm{c}} \bullet \mathrm{kg}^{-1}$, en relación a la concentración inicial.

De igual forma, el comportamiento observado en la concentración de Ca coincide con lo reportado por Combatt et al. (2008) y Combatt et al. (2009) quienes evidenciaron incrementos en este elemento después del encalamiento, debido a la eficiencia de disolución de la calcita al producir iones de $\mathrm{Ca}^{2+}$ y aumento en su disponibilidad por incremento del $\mathrm{pH}$ en el suelo (Navarro y Navarro 2003), como consecuencia del aporte de iones $\mathrm{OH}^{-}$que reducen la acidez (Combatt et al. 2007). Sadzawka y Campillo (1993) indican que el carbonato de Ca se disuelve a medida que los iones hidróxido son removidos de la solución del suelo, lo cual produce iones $\mathrm{Ca}^{2+}$ y bicarbonato. De la misma forma, Kamprath (1967) y Zetina et al. (2005) afirman que la práctica del encalado al neutralizar el aluminio, hierro y manganeso intercambiables, aumenta los contenidos de las bases y su porcentaje de saturación.

De acuerdo con Franchini et al. (1999), el aumento en la concentración de $\mathrm{Mg}$ que se evidenció con la incorporación de abono verde y siembra de $B$. rapa, posiblemente se debe a la generación de ácidos orgánicos de Ca y Mg por parte del nabo forrajero, los cuales repercuten en el mantenimiento de estos elementos en la solución del suelo; de forma similar, Muhrizal et al. (2003) observaron una correlación directa entre la composición química de estos materiales vegetales y los cationes básicos presentes en la fase sólida y soluble de SSA en Malasia. De la misma manera, Arias (2011) encontró un mayor incremento de $\mathrm{Mg}$ en el suelo después 120 días de la siembra de abono verde. Asimismo, la adición de cal dolomita induce el desplazamiento del elemento hacia la fase intercambiable y promueve su disponibilidad por el incremento del pH (Navarro y Navarro 2003; Zetina et al. 2005; Combatt et al. 2009).

Por su parte, el mayor contenido de $\mathrm{K}$ que se evidenció con la incorporación de abono verde, posiblemente se debe a la liberación de ácidos orgánicos en forma de sales $\left(\mathrm{K}^{+}, \mathrm{Ca}^{2+} \mathrm{y}\right.$ $\left.\mathrm{Mg}^{2+}\right)$, a través del proceso de mineralización del nabo forrajero (Franchini et al. 1999; Damatto et al. 2006; Demanet y Canales 2008). Estos resultados concuerdan con Navarro y Navarro (2003) quienes afirman que la adición de materia orgánica incrementa la fijación del $\mathrm{K}^{+}$a los coloides del suelo. El aporte de bases 
intercambiables por parte de las enmiendas orgánicas (abonos verdes), de acuerdo con Lazcano (1996), favorece la adsorción de este elemento por los coloides, evita la lixiviación y permite la liberación del $\mathrm{K}^{+}$atrapado dentro de las arcillas. Debido a que el $\mathrm{Ca}^{2+}$ al tener un volumen mayor que el potasio, cuando se introduce entre los espacios interlaminares y los separa permitiendo la salida del $\mathrm{K}^{+}$(Navarro y Navarro, 2003). Igualmente, Hernández y Viteri (2006) evidenciaron un aumento en la disponibilidad de este elemento con la aplicación de 12,5 t• ha-1 de cal $(\mathrm{CaO})$ y Zetina et al. (2005) señalaron que el suelo encalado con cal dolomita mostró mayor contenido de $\mathrm{Ca}^{2+}, \mathrm{Mg}^{2+}$ y $\mathrm{K}^{+}$intercambiables y a su vez, disminuyó el porcentaje de saturación de $\mathrm{Al}^{3+}$ intercambiable.

Por otro lado, se observó que la aplicación de los tratamientos no influyó en la concentración del $\mathrm{Na}$, dado que no se presentaron diferencias significativas; sin embargo, Gómez et al. (2005) reportaron que con la aplicación de 12,5 t• ha-1 de $\mathrm{Ca}(\mathrm{OH})_{2}+10$ t•ha-1 de materia orgánica se redujo la concentración de $\mathrm{Na}$, lo cual no se evidenció en este estudio.

En cuanto a la respuesta del cultivo indicador, la variable altura de la planta presentó diferencias estadísticamente significativas ( $p>0.05)$, donde el valor más alto se registró con el tratamiento de incorporación de microorganismos eficientes, seguido por el agronómico, en comparación al testigo que generó el valor más bajo (Figura 2A). La altura obtenida en las plantas del tratamiento con microorganismos eficientes, pudo deberse probablemente a que esta técnica acelera la descomposición de materiales orgánicos en el suelo y conduce a una mayor liberación de los nutrientes necesarios para el crecimiento de las plantas (Limanska et al. 2013). De igual forma, Hernández et al. (2005) afirman que el componente microbiológico del suelo influye de manera directa en la elongación de raíces y tallos, atribuida a sustancias de tipo hormonal estimuladoras de crecimiento.

El efecto del tratamiento testigo sobre la altura de las plantas, posiblemente se deba a que no se realizó una corrección de las condiciones químicasdel suelopormediodelaincorporación de materiales encalantes u orgánicos. Porque las características particulares de los SSA, como el $\mathrm{pH}$ extremadamente ácido y las altas concentraciones de $\mathrm{Al}$, entre otras, influyen negativamente en el crecimiento de las plantas, tal como lo indican Samac y Tesfaye (2003), quienes manifiestan que los ápices de las raíces son sensibles a la presencia de $\mathrm{Al}$ en el suelo, lo cual causa inhibición del crecimiento, limita la toma de agua y nutrientes y afecta la productividad de las mismas (Samac y Tesfaye 2003; Casierra y Cárdenas 2007).

Por su parte, los tratamientos correspondientes a la adición de bagacillo, siembra de $B$. rapa L. como forraje y la incorporación del mismo como abono verde, presentaron una respuesta positiva, lo cual concuerda con Gómez (2007) y Cuaresma y Valdez (2009), quienes alcanzaron la mayor altura en plantas de $B$. oleracea mediante la siembra en sustratos compuestos por compost y paja de arroz. Sin embargo, los resultados obtenidos en la variable altura de $B$. oleracea en los seis tratamientos evaluados en este estudio, son inferiores a los reportados por Vázquez et al. (2004) quienes indican que esta especie, en condiciones de crecimiento 
normales, alcanza alturas entre $50-70 \mathrm{~cm}$, mientras que la máxima registrada en este estudio fue de $32,2 \mathrm{~cm}$.

En cuanto al diámetro de la cabeza del cultivo indicador, se presentaron diferencias estadísticas significativas entre el testigo y los tratamientos ( $p>0.05$ ) (Figura 2B). Los valores medios alcanzados con las metodologías evaluadas fueron similares, excepto en el testigo; sin embargo, se registró la media más alta con la siembra de $B$. rapa L. como forraje, seguido por la incorporación de esta especie como abono verde. Asimismo, los tratamientos correspondientes a la adición de bagacillo de caña, la aplicación de microorganismos eficientes y el agronómico, mostraron resultados positivos respecto al testigo, en el cual no se pudo medir esta variable, puesto que la planta se afectó en su crecimiento y desarrollo al no recibir corrección en el suelo. Los resultados obtenidos con el tratamiento de siembra de B. rapa L., probablemente se debe a que la plantación previa de esta especie benefició el establecimiento del cultivo indicador, ya que según Birbaumer et al. (2000) el crecimiento de esta especie favorece el reciclaje de nutrientes, especialmente fósforo y nitrógeno.

En general, la respuesta de los tratamientos evaluados, de acuerdo con Knox et al. (2000), Lazcano (2001), Díaz y Lagos (2009) y Osorno (2012), pudo deberse a que ciertos cultivos son más tolerantes a la acidez del suelo que otros, al aumento en la cantidad de Ca y Mg generada por la adición del material encalante

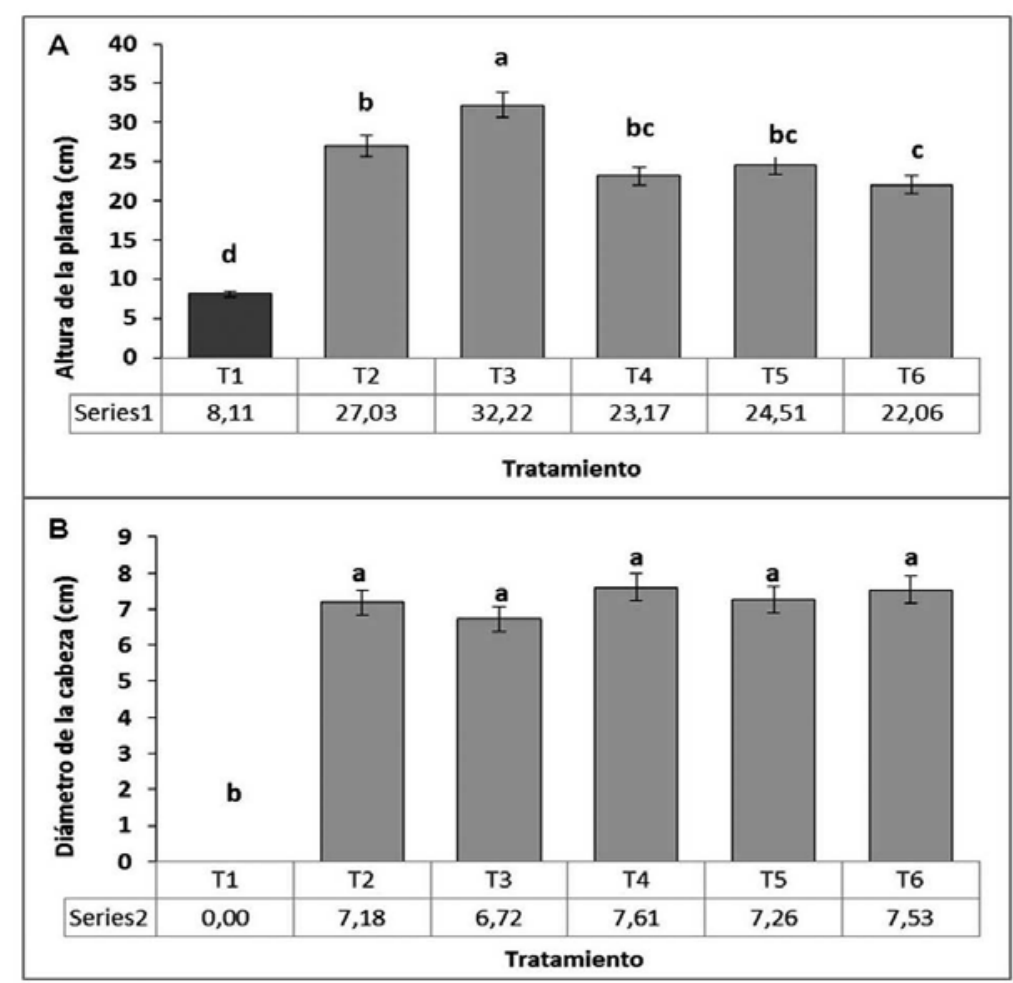

Figura 2. Medición de variables agronómicas: A. Altura de la planta y B. Diámetro de la cabeza del coliflor (B. oleracea) en un suelo sulfatado ácido (SSA). T1: Testigo absoluto; T2: Tratamiento químico; T3: Aplicación de microorganismos eficientes; T4: Siembra de B. rapa L. como forraje; T5: Adición de bagacillo de caña; T6: Incorporación de B. rapa L. como abono verde. Promedios seguidos de letras distintas en la misma serie presentan diferencias significativas según la prueba de Tukey $(5 \%)$. 
y al incremento del $\mathrm{pH}$, lo cual favorece la absorción de agua y nutrientes, además permite el establecimiento de las plantas.

De igual forma, el uso complementario de la cal en combinación con técnicas como la siembra de especies vegetales como forraje o cobertura, incorporación de abonos orgánicos o la adición de microorganismos eficientes, probablemente contribuyó con la obtención de esta respuesta, ya que de acuerdo con Molina (2008) estas metodologías constituyen prácticas apropiadas para corregir problemas de acidez y a su vez, favorecen la conservación y uso del suelo. Sin embargo, bajo condiciones de crecimiento en suelos normales, Jaramillo y Díaz (2006) reportaron que el tamaño de las pellas de $B$. oleracea alcanzan diámetros de 15 - $30 \mathrm{~cm}$, superiores a los obtenidos en este estudio, donde el mayor promedio fue de $7,6 \mathrm{~cm}$, siendo muy baja en comparación con dicho reporte.

\section{CONCLUSIONES}

La siembra de B. rapa L. como forraje y su incorporación como abono verde, junto con el uso complementario de enmiendas calcáreas como correctivo, indujo a un aumento en las concentraciones de $\mathrm{Ca}, \mathrm{Mg}$ y $\mathrm{K}$ en el suelo sulfatado ácido, respecto a las metodologías evaluadas bajo condiciones de invernadero.

La aplicación de microorganismos eficientes en las raíces de las plántulas de coliflor (Brassica oleracea var. Botrytis), antes de su siembra, generó la mejor respuesta en la variable altura, en comparación con las técnicas evaluadas.

Se recomienda la implementación de este estudio en campo para corroborar la respuesta de los tratamientos evaluados, debido a que su implementación a nivel de invernadero no asegura el mismo comportamiento, por las diferentes condiciones climáticas u otros factores de alteración que puedan presentarse.

\section{AGRADECIMIENTOS}

Los autores agradecen a la Dirección de Investigaciones - DIN de la Universidad Pedagógica y Tecnológica de Colombia UPTC Tunja, por el apoyo financiero para la ejecución del proyecto de investigación, el cual dio origen al presente artículo.

\section{REFERENCIAS}

Arias, F. 2011. Determinación del efecto de la incorporación deenmiendas orgánicas más abono verde en los niveles de fertilidad del suelo en el lugar de acumulación de lodos de perforación petrolera Pozo Guanta 01. Tesis Ingeniero Agrónomo, Escuela Superior Politécnica de Chimborazo, Riobamba - Ecuador. 103 p.

\section{Birbaumer, G. y Grupos temáticos del} proyecto. 2000. Cultivar sin arar. Labranza mínima y siembra directa. Proyecto de Conservación de Suelo y Agua en la Zona Andina - Proyecto Checua, Bogotá, Colombia. 146 p.

\section{Bernal, M., Sánchez, M., Paredes C. and Roig,} A. 1998. Carbon mineralization from organic wastes at different composting stages during their incubation with soil. Agriculture, Ecosystems \& Environment. 69(3): 175-189. 
Cabrales, E. 2007. Dinámica nutrimental y caracterización microbiológica de los suelos sulfatados ácidos del valle del Sinú - Colombia. Línea de Investigación Suelos y aguas, Grupo de Investigación Manejo de cultivos tropicales, Facultad de Ciencias Agrarias, Universidad de Córdoba, Córdoba. 201 p.

Casierra, F. y Cárdenas, J. 2007. Influencia del aluminio sobre el crecimiento de la raíz en Coliflor (Brassica oleracea L., var. Botrytis, Hib. 'Nevada F1'). Revista U.D.C.A. Actualidad \& Divulgación Científica. 10(1): 149-157.

Castro, H. y Munevar, O. 2011. Mejoramiento químico integral de suelos ácidos mediante el uso combinado de materiales encalantes. XIX Congreso Venezolano de la Ciencia del Suelo. Sociedad Venezolana de la Ciencia del Suelo, Calabozo, Venezuela, noviembre de 2011.

Combatt, E., Cabrales, E., Cardona, C., Jarma, A., y Martínez, G. 2007. Caracterización de suelos sulfatados ácidos y la respuesta de tres especies vegetales en el bajo Sinú de Córdoba. Grupo de Investigación en Cultivos Tropicales de Clima Cálido, Oficina Administrativa de Investigación y Extensión, Universidad de Córdoba. Córdoba. 260 p.

Combatt, E., Martínez, Z., Cabrales, E., Martínez, G., Castillo, C. y Palencia, M. 2004. Caracterización fisicoquímica y mineralógica de los suelos sulfatados ácidos en el transecto San Carlos Cotorra- Carrillo. Grupo de Investigación en Cultivos Tropicales de Clima Cálido, Universidad de Córdoba. Córdoba. 3 p.

Combatt, E., Jarma, A. y Maza, L. 2008. Crecimiento de Brachiaria decumbens Stapf y Cynodon nlemfuensis Vanderyst en suelos sulfatados ácidos de Córdoba. Revista MVZ Córdoba 13(2): 1380-1392.

Combatt, E., Mercado, T. y Palencia, G. 2009. Alteración Química de la solución de un suelo sulfatado acido, con encalamiento y lavado en columna disturbadas. Revista U.D.C.A. Actualidad $\varepsilon$ Divulgación Científica. 12(1): 101-111.

Cuaresma, J. y Valdez, M. 2009. Effects of substrate volume on the growth of cabbage (Brassica oleracea L.) plug transplants. USM R \& D Journal 17(2): 171-177.

Dalzell, H., Roddlestone, A., Gray, K. y Thurairajan, K. 1991. Manejo del suelo: producción y uso del composte en ambientes tropicales y subtropicales. Boletín de suelos de la FAO 56, Organización de las Naciones Unidas para la Agricultura y la Alimentación, Roma. 178 p.

Damatto, E., Lyra R., Leonel S. y Fernandes D. 2006. Alterações em propriedades de solo adubado com doses de composto orgânico sob cultivo de bananeira. Revista Brasileira de Fruticultura, Jaboticabal. 28 (3): 546-549.

Demanet, R. y Canales, C. 2008. Establecimiento, cultivo y producción de nabo forrajero, suplemento alimenticio 
de buenas perspectivas.http:// lacteos.watts.cl/images/Img_Editor/ DocAdjuntos/Nabos\%20Forrajeros.pdf [8 Agosto 2011].

Dent, D. y Dawson, B. 2000. The acid test. An expert system for acid sulphate soils. This manual is intended to accompany the Evaluation edition (version 0.9) of the Acid Sulphate Soils Identikit. 36 p.

Díaz, O., Montenegro, D. y Lagos, J. 2009. Acción de microorganismos eficientes sobre la actividad de intercambio catiónico en plántulas de acacia (Acacia melanoxylon) para la recuperación de un suelo del municipio de Mondoñedo, Cundinamarca. Colomb. For. 12(1): 141-160.

Ercoli, E., Gálvez, J., Di Paola, M., Cantero, J., Videla, S. y Medaura, C. 1999. Biorremediación de suelos altamente contaminados. Ingepet. Expl-8-EE-03, Perú. 11 p.

Franchini, J. Miyazawa M., Pavan M. e Malavolta, E. 1999. Dinâmica de íons em solo ácido lixiviado com extratos de resíduos de adubos verdes e soluções puras de ácidos orgánicos. Pesq. Agropec. Bras, Brasilia. 34(12): 2267-2276.

\section{GISSAT - Grupo Interinstitucional de} Investigación en Suelos Sulfatados Ácidos Tropicales. 2006. Proyecto Colciencias - UPTC, Caracterización de la problemática de suelos sulfatados ácidos improductivos y evaluación del manejo para su habilitación agrícola. Distrito Riego del Alto de Chicamocha
(Boyacá). Universidad Pedagógica y Tecnológica de Colombia, Tunja. 376 p.

Gómez, C. 2007. Respuesta de la coliflor (Brassica oleracea var. botrytis) a la aplicación de tres fuentes y cuatro niveles de abonos orgánicos en Quiroga Imbabura. Tesis Ingeniero Agropecuario, Universidad Técnica del Norte, Ibarra (Ecuador) $132 \mathrm{p}$.

Gómez, M., Castro, H. y Pacheco, W. 2005. Recovery and management of actual acid sulphate soils in Boyacá (Colombia). Agronomía Colombiana. 23(1): 128-135.

González, M. 2005. Recuperación de suelos contaminados con metales pesados utilizando plantas y microorganismos rizosféricos. Terra Latinoamericana. 23(1): 29-37.

Hernández, D.yCastro, H. 2007. Rehabilitación agrícola de suelos sulfatados ácidos en el altiplano Cundiboyacense. Memorias III Encuentro Nacional de Agricultura y Conservación, junio de 2007. 20p.

Hernández, D. y Viteri, S. 2006. Selección de abonos verdes para el manejo y rehabilitación de los suelos sulfatados ácidos de Boyacá (Colombia).Agronomía Colombiana. 24(1): 131-137.

Hernández, M., García, C., Pascual, J. y Hernández, M. 2005. Posible efecto fitohormonal de las bacterias fijadoras de nitrógeno. Revista Agropecuaria. 870: 62-66. 
Honorato, R. y Bonomelli, C. 2002. Suelos Degradados y Agricultura Sustentable. Agronomía y Forestal. UC (15): 20-24.

\section{IGAC - Instituto Geográfico Agustín Codazzi.} 2006. Métodos analíticos de laboratorio de suelos. Sexta edición, Imprenta Nacional, Bogotá. D.C. 648 p.

Jaramillo, N. y Díaz, D. 2006. El Cultivo de las Crucíferas. Manual Técnico Corporación Colombiana de Investigación Agropecuaria - CORPOICA, Centro de Investigación La Selva,Rionegro, Antioquia, Colombia. 176 p.

Kamprath, E.1967. Acidez del suelo y su respuesta al encalado. International Soil Testing.Raleigh, North Carolina. p15-24.

\section{Knox,A., Seaman, J.,Adriano, D. and Pierzynski,} G., 2000. Chemophytostabilization of metals in contaminated soils.En: Wise, D., Trantolo, D., Cichon, E., Inyang, H. y Stottmeister, U., (Ed). Bioremediation of contaminated soils.Marcel Dekker, Inc., New York. p811-836.

Lazcano, I. 1996. Controle la acidez y alcalinidad y aumente la fertilidad de su suelo. Informaciones Agronómicas. 1(4): 1-16.

Lazcano, I. 2001. Cal agrícola: conceptos básicos para la producción de cultivos. International Plant Nutrition Institute IPNI. http://www.ipni.net/ppiweb/iamex. nsf/\$webindex/5057DEAFC8DE54CC06 256AD1005D7CB9/\$file/cal+agricola+c onceptos+basicos+para+la+produccion + de+cultivos.pdf [10 Octubre 2012].
Limanska, N., Ivanytsia, T., Basiul, O., Krylova, K., Biscola, V., Chobert, J., Ivanytsia, V. and Haertlé, T. 2013. Effect of Lactobacillus plantarum on germination and growth of tomato seedlings. Acta Physiologiae Plantarum. 35(5): 1587-1595.

López, R. 2002. Degradación del suelo: causas, procesos, evaluación e investigación. Segunda edición. Talleres gráficos del Centro Interamericano de Desarrollo e Investigación Ambiental y Territorial Universidad de los Andes (CIDIAT), Mérida (Venezuela). p40-75.

Molina, E. 2008. Acidez de suelo y encalado. Tomado de Molina, E. 1998. Encalado para la corrección de la acidez del suelo. ACCS. San José, Costa Rica. http://anfacal. org/media/Biblioteca_Digital/Agricultura/ Neutralizacion_de_Suelos_Acidos/JMencalado_y_acidez.pdf [5 Mayo 2014].

Montaño, J. y Forero, F. 2013. Efecto de subproductos orgánicos del proceso panelero sobre propiedades físicas de un suelo sulfatado ácido. Corpoica Cienc. Tecnol. Agropecu. 14(2): 207-214.

Muhrizal, S., Shamshuddin, J., Husni, M. H. A., and Fauziah, I. 2003. Alleviation of aluminum toxicity in an acid sulfate soil in Malaysia using organic materials. Communications in soil science and plant analysis. 34(19-20): 2993-3011.

Navarro, S. y Navarro, G. 2003. Química Agrícola. El suelo y los elementos químicos esenciales para la vida vegetal. Segunda edición. Mundi-Prensa, Madrid. 487p. 
Ocampo, J., Chavarriaga, W. y Ceballos, N. 2007. Valoración de tres fuentes de calcio en suelos ácidos de la granja tesorito y la respuesta en producción para el cultivo de arveja. Agron. 15(2): 7-15.

Osorno, H. 2012. Mitos y realidades de las cales y enmiendas en Colombia. Trabajo de grado, Universidad nacional de Colombia, Medellín, Colombia. 70p.

Plata, A., Forero F., Balaguera H. y Serrano P. 2009. Evaluación del efecto de la aplicación de cachaza fresca al cultivo de repollo (Brassica oleraceae var. capitata L.). Ciencia y Agricultura. 7 (1): 29-42.

Prasad, K., Kumar, N. and Sharma, S. 2010. Bioremediation: developments, current practices and perspectives. Genetic Engineering and Biotechnology Journal. 2010 (GEBJ - 3): 1-20.

Sadzawka A. y Campillo R. 1993. Problemática de la acidez de los suelos de la IX región. IPA Carillanca. 12 (3): 3-12.

Samac, D. and Tesfaye M. 2003. Plant improvement for tolerance to aluminum in acid soils - a review.Plant Cell, Tissue and Organ Culture. 75(3): 189-207.
Taylor, G., McDonald, J., Hunter, D., Bertsch, P., Elmore, D., Rengel, Z. and Reid, R. 2000. Direct measurement of aluminum uptake and distribution in single cells of Characorallina. Plant Physiol. 123: 987-996.

Urquiza, M. 2002. Manejo sostenible de los suelos. Compendio. http://www. actaf.co.cu/index.php?option $=\mathrm{com}_{-}$ mtree\&task=att_download\&link_ id=122\&cf_id=24 [15 Septiembre 2011].

Vázquez, M., Piro, M. y Lanfranco, J. 2004. Corrección de suelos ácidos de la Pampa húmeda para la producción de alfalfa. Informaciones Agronómicas. 23: 1-12.

Viteri, S. y Velandia, J. 2006. Evaluación de asociaciones vegetales por su potencial como fuente de materia orgánica para los suelos de Samacá (Boyacá). Agron. Colomb. 24(1): 138-146.

Zetina, R.,Trinidad, A., Opereza, J., Volke, V. y Landois, L. 2005. Relación bases intercambiables - rendimiento de maíz en un cambisol dístrico con labranza, encalado y abono verde. Terra Latinoamericana. 23 (3): 389-397.

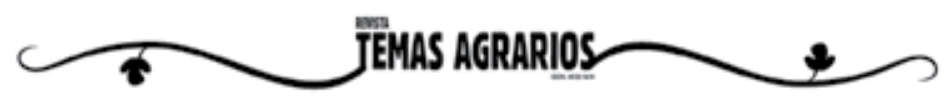

